\title{
Technique and rationale for branch-first total aortic arch repair
}

\author{
Michelle Kim, MBBS, and George Matalanis, MBBS, MS, FRACS
}

\begin{abstract}
Objective: Our objective was to describe the technique and rationale for branchfirst total aortic arch repair.

Methods: Branch-first total aortic arch repair involves serial clamping, reconstruction, and reperfusion of each of the arch branches using a specially designed trifurcation graft with a side port. During this sequence, perfusion to the heart and distal organs are preserved and continuous antegrade cerebral perfusion is permitted via the trifurcation graft. The diseased aorta is excised and replaced with a Dacron graft (W.L. Gore and Associates, Newark, Del) with a perfusion side port. The trifurcation graft is anastomosed to the new proximal ascending aorta.
\end{abstract}

Results: The branch-first technique permits total aortic arch repair without global cerebral circulatory arrest and excessive hypothermia. It shortens distal organ and cardiac ischemic time, and reduces the opportunity for air and particulate embolization during aortic repair.

Conclusions: Branch-first total aortic arch repair allows continuous antegrade cerebral perfusion and shortens distal organ and cardiac ischemic time, with unobstructed access to the full extent of the diseased aortic arch. (JTCVS Techniques 2020;4:1-4)

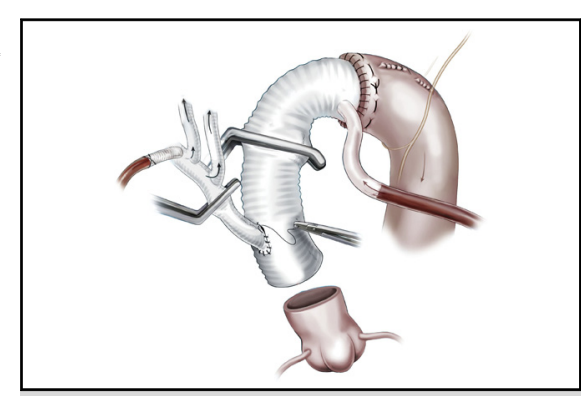

Branch-first total arch repair using continuous antegrade cerebral perfusion.

\section{CENTRAL MESSAGE}

Arch branches are reconstructed using a trifurcation graft without interrupting cardiac and distal perfusion. The perfusion port enables continuous cerebral perfusion during arch reconstruction.
The branch-first technique permits total aortic arch repair without global cerebral circulatory arrest and excessive hypothermia. ${ }^{1,2}$ It has been used successfully in both the elective and emergency settings. The concept involves serial clamping, reconstruction, and reperfusion of each of the

From the Department of Cardiac Surgery, Austin Health, Melbourne, Australia. Accepted for the American Association for Thoracic Surgery Aortic Symposium 2020.

Received for publication Aug 26, 2020; accepted for publication Sept 17, 2020; available ahead of print Sept 22, 2020.

Address for reprints: Michelle Kim, MBBS, Department of Cardiac Surgery, Austin Health, 145 Studley Road, Heidelberg, Melbourne, Victoria, Australia (E-mail: mkim1389@gmail.com).

2666-2507

Copyright (C) 2020 The Authors. Published by Elsevier Inc. on behalf of The American Association for Thoracic Surgery. This is an open access article under the CC BY-NCND license (http://creativecommons.org/licenses/by-nc-nd/4.0/).

https://doi.org/10.1016/j.xjtc.2020.09.014

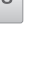




\section{Abbreviations and Acronyms \\ IA $=$ innominate artery \\ LCCA $=$ left common carotid artery \\ LSCA $=$ left subclavian artery \\ TAPP $=$ trifurcation arch graft with side perfusion port}

core cooling to $28^{\circ} \mathrm{C}$. If distal body circulatory arrest is anticipated, then cooling is extended to $25^{\circ} \mathrm{C}$. After institution of bypass, the IA is clamped just proximal to its bifurcation and then just distal to its origin from the arch and divided. The distal IA stump is anastomosed to the first limb of the trifurcation graft with 5/0 Prolene suture. Thorough de-airing maneuvers are performed and then the clamps are rearranged to resume antegrade perfusion to the IA via a separate head circuit that attaches to the side port of the TAPP graft (Figure 1). The IA proximal stump is often conveniently rapidly ligated with a silk suture.

The essential detail relevant to the preservation of right hemispheric flow during the innominate debranching sequence is the clamp location. This arrangement is quite different from that of carotid endarterectomy, where the distal common and external carotid arteries are clamped, and there is sole reliance on an intact circle of Willis for the sole major collateral supply. The very proximal position of the IA clamp allows the rich collateral networks around the head and neck, fed from ongoing perfusion of the other 2 arch branches and returning to the right carotid via its external carotid branch, to augment right hemispheric perfusion over and beyond that provided by the circle of Willis. Furthermore, typically only short clamp times are required, in contrast with unilateral antegrade perfusion where the same collateral network is utilized but the exclusion time is for the whole duration of arch reconstruction.

With the debranched IA out of the field, there is further improvement in exposure to the LCCA. The LCCA can be mobilized, reconstructed, and reperfused in the same sequence to the second limb of the TAPP graft. Again, the same rich network is utilized to maintain left hemispheric perfusion for the even shorter clamp time for this smaller anastomosis (Figure 2).

If there is adequate exposure to the left subclavian artery (LSCA) it is mobilized and clamp isolated, with the assistant helping to ease it out of the depth of the surgical field. This is reconstructed and reperfused in the same sequence to the third limb of the TAPP graft. Throughout the debranching process, perfusion is not interrupted to the heart or distal organs. The branched trifurcation graft is laid out of the operative field and is utilized for continuous antegrade cerebral perfusion throughout the operation. Throughout the debranching and reperfusion sequence for each of the head vessels, the head pump is adjusted

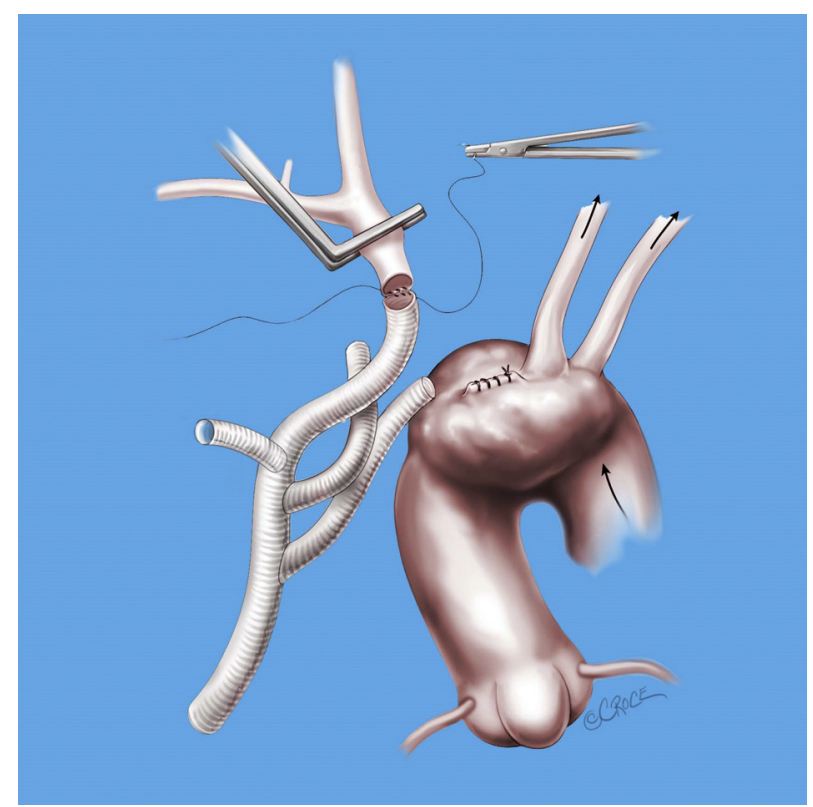

FIGURE 1. The innominate artery (IA) is clamped just proximal to its bifurcation and then just distal to its origin from the arch and divided. The distal IA stump is anastomosed to the first limb of the trifurcation graft with 5/0 Prolene suture. Thorough de-airing maneuvers are performed and then the clamps are rearranged to resume antegrade perfusion to the IA via a separate head circuit, which attaches to the side port of the trifurcation arch graft with side perfusion port (TAPP) graft. The IA proximal stump is often conveniently rapidly ligated with a silk suture. The very proximal position of the IA clamp allows the rich collateral networks around the head and neck to augment right hemispheric perfusion over and beyond that provided by the circle of Willis.

for cerebral perfusion that maintains flow independent of the rest of the body. A median sternotomy is performed. Division of the innominate vein tributaries allows it to be retracted easily without recourse to sacrificing the vein, thereby preserving left hemispheric venous drainage. The thymic fat pad is divided in the midline but preserved for the completion stage, when it is reconstituted as a biological protection for the vein and Dacron material (W.L. Gore and Associates, Newark, Del) in case of future surgery.

\section{Branch-First Reconstruction}

The innominate artery (IA) and left common carotid artery (LCCA) are carefully exposed before institution of cardiopulmonary bypass. Care is exercised to avoid excessive retraction of the aorta and its branches to reduce the risk of atheroembolization. An appropriately sized Dacron trifurcation graft with a perfusion side port (TAPP) (TAPP Graft; Vascutek Ltd, Renfrewshire, Scotland, United Kingdom) (Figure 1, left) is selected and the branched limbs are aggressively trimmed to the appropriate length. A connector is tied to the side port for ease and rapidity of subsequent connection to the arterial circuit.

Cardiopulmonary bypass is initiated using femoral arterial cannulation and right atrial venous cannulation, and 


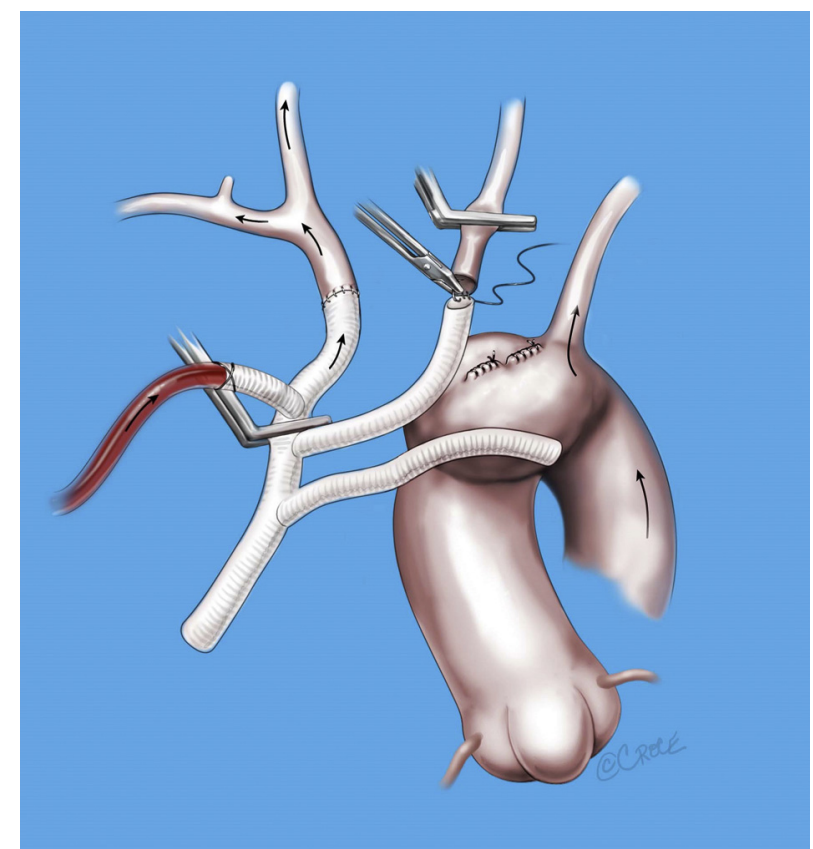

FIGURE 2. With the debranched innominate (IA) out of the field, there is further improvement in exposure to the left common carotid artery (LCCA). The LCCA is mobilized, divided between 2 clamps, and anastomosed to the second limb of the trifurcation graft with 5/0 Prolene suture. Throughout this time, antegrade cerebral perfusion continues via the side port of the trifurcation arch graft with side perfusion port (TAPP) graft. Thorough de-airing maneuvers are performed and then the clamps are again rearranged to resume antegrade perfusion to both the IA and LCCA.

progressively to maintain a mean pressure of 50 to $60 \mathrm{~mm}$ $\mathrm{Hg}$ in the radial arterial line, with the flows increasing from 500 to $1000 \mathrm{~mL} / \mathrm{min}$. The temperature of the head circuit is targeted at the initial core cooling target.

Again with the debranching of the IA and LCCA, there is greatly improved exposure to the more deeply lying LSCA. Nonetheless, there are patients where the LSCA remains difficult to reach. There are a number of maneuvers that can be employed to assist. Even a short extension of the skin incision into the neck along the anterior border of the left sternocleidomastoid muscle can result in greatly improved exposure. Secondly, with cerebral perfusion ensured by flow from the dedicated head pump, selectively decreasing distal perfusion pressure can decompress the aorta to generate more space to access the LSCA. Another option is to delay reconstruction of the LSCA until after institution of distal circulatory arrest or proximal descending clamping, when a lot of space is created by the total decompression of the distal arch.

\section{Aortic Arch Replacement}

Once the debranching is complete, the descending aorta is controlled either with a clamp, endoluminal balloon or distal circulatory arrest, depending on pathology treated

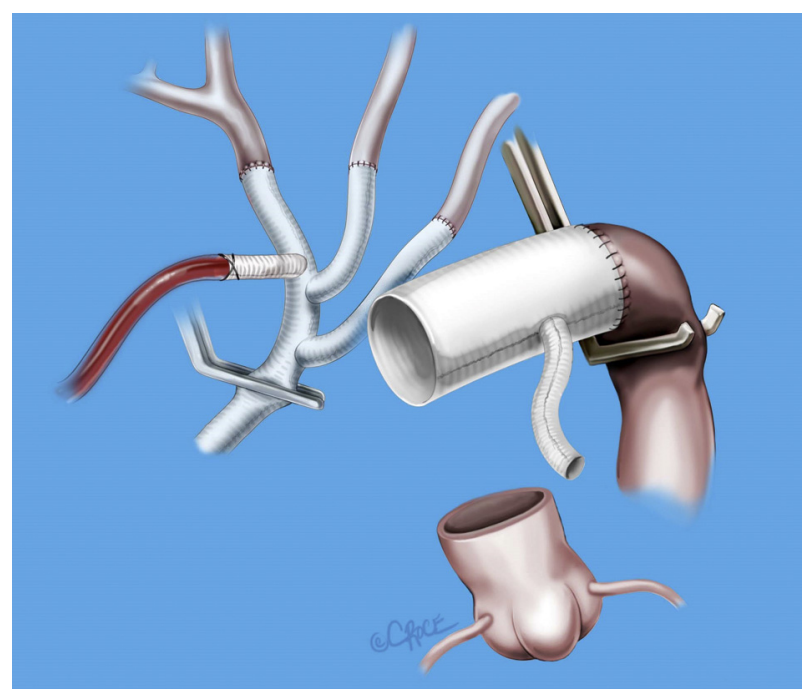

FIGURE 3. The descending aorta is controlled either with a clamp, endoluminal balloon or distal circulatory arrest, depending on pathology treated and local condition. Myocardial protection is initiated and maintained by antegrade and retrograde cardioplegia. Visceral and spinal cord perfusion is maintained via femoral arterial inflow. Antegrade cerebral perfusion continues via the side port of the trifurcation arch graft with side perfusion port (TAPP) graft to the attached head vessels. The arch is excised and the distal aortic anastomosis is performed using a tube graft with a pre-attached side port for subsequent antegrade distal perfusion. De-airing maneuvers are performed and the aortic clamp is repositioned on the tube graft proximal to the side port allowing distal re-perfusion to be swapped from femoral retrograde to antegrade.

and local conditions. In cases where the distal aorta is clamped, visceral and spinal cord perfusion is maintained via femoral arterial inflow. The heart is now excluded from the circulation for the first time and myocardial protection is initiated and maintained by antegrade and retrograde cardioplegia.

The ascending aorta and arch remnant are excised and an appropriately sized graft is anastomosed to the distal aorta (Figure 3). Either a Dacron graft (Ante-Flo; Vascutek Ltd) or a frozen elephant trunk graft (Thoraflex Hybrid; Vascutek Terumo, Inchinnan, Scotland, United Kingdom; or EVita Hybrid; Jotec GmbH, Hechingen, Germany) is used. A preattached side port on the main graft (as in the AnteFlo versions of the regular or Thoraflex grafts) is ideal for subsequent antegrade body perfusion, otherwise direct cannulation of the graft is required. De-airing maneuvers are performed and the aortic clamp is repositioned on the tube graft proximal to the side port or perfusion cannula allowing distal reperfusion to be swapped from femoral retrograde to antegrade.

The common stem of the TAPP graft is passed under the innominate vein and anastomosed to a hole in the tube graft corresponding to the new ascending aorta in an end-to-side fashion (Figure 4). Note that cerebral perfusion is 


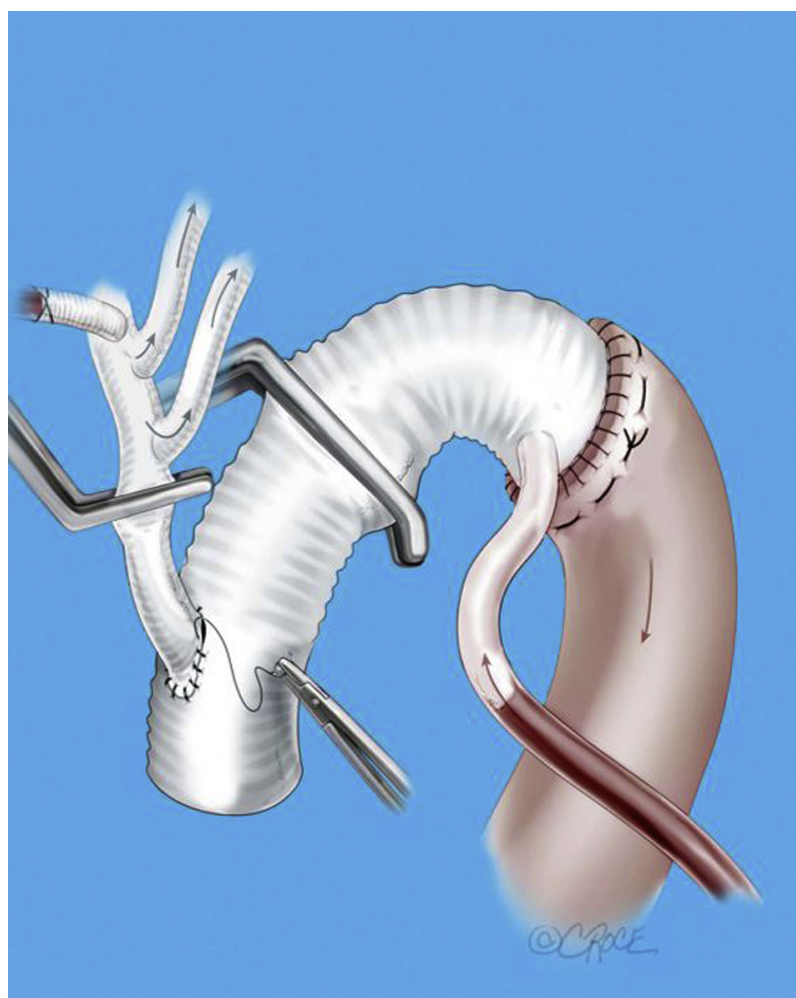

FIGURE 4. The common stem of the trifurcation arch graft with side perfusion port (TAPP) graft is passed under the innominate vein and anastomosed to a hole in the tube graft corresponding to the new ascending aorta. Cerebral perfusion is maintained throughout this period as the side port of the TAPP graft is conveniently located close to the innominate (IA) branch. This anastomosis can be delayed until after the proximal aortic anastomosis to further reduce myocardial ischemia time.

maintained throughout this period because the side port of the TAPP graft is conveniently located close to the IA branch. This anastomosis can be delayed until after the proximal aortic anastomosis to reduce myocardial ischemia time further. It is important that the site of attachment of TAPP graft on the ascending graft is sufficiently proximal to the distal aortic anastomosis to allow an adequate zone for potential future endovascular procedures.

\section{CONCLUSIONS}

The rationale for the branch-first total aortic repair technique can be summarized in terms of the main components of the procedure as described above. Firstly, the reconstruction and reperfusion sequence of the arch branches allows cerebral perfusion to be secured early during the operation and isolated in a separate head circuit, which is especially important in cases of acute aortic dissection with malperfusion. It therefore avoids total cerebral circulatory arrest, shortens distal organ and cardiac ischemic time, and reduces the opportunity for air and particulate embolization during aortic repair. Subsequently, the aortic repair can be performed with unobstructed access to the full extent of the aorta with continuous cerebral and distal perfusion.

\section{Conflict of Interest Statement}

The authors reported no conflicts of interest.

The Journal policy requires editors and reviewers to disclose conflicts of interest and to decline handling or reviewing manuscripts for which they may have a conflict of interest. The editors and reviewers of this article have no conflicts of interest.

\section{References}

1. Matalanis G, Perera NK, Galvin SD. Aortic arch replacement without circulatory arrest or deep hypothermia: the branch-first technique. J Thorac Cardiovasc Surg. 2015;149:S76-82.

2. Matalanis G, Koirala RS, Shi WY, Hayward PA, McCall PR. Branch-first aortic arch replacement with no circulatory arrest or deep hypothermia. J Thorac Cardiovasc Surg. 2011;142:809-15.

3. Galvin SD, Matalanis G. Continuous perfusion "Branch-first" aortic arch replacement: a technical perspective. Ann Cardiothorac Surg. 2013;2:229-34.

Key Words: total aortic arch repair, aortic arch replacement, antegrade cerebral perfusion, branch-first 